'A Posod, 'Y Krepp, 'V Neubauer, 'K Wegleiter, 'M Urbanek, 1,2M Keller, 'U KiechlKohlendorfer, 'E Griesmaier. 'Department of Paediatrics IV, Division of Neonatology, Innsbruck Medical University, Innsbruck, Austria; 'Department of Paediatrics, Children's Hospital Passau, Passau, Germany

Background Supraphysiologic oxygen concentrations are toxic to the developing brain. Inflammatory processes increase the risk of brain injury. We have previously shown a protective effect of dextromethorphan, a NMDA receptor antagonist and sigma-1 receptor $(\sigma 1 \mathrm{R})$ agonist, in an animal model of hyperoxia-induced neonatal brain injury. In adult brain injury, sigma agonists have a proven therapeutic potential.

Aim To assess the highly selective $\sigma 1 \mathrm{R}$ agonist PRE-084 in a newborn animal model of inflammation-sensitized hyperoxia-induced brain injury.

Methods Rat pups were randomly pre-sensitized with a single intraperitoneal (ip) injection of i) LPS or ii) vehicle on postnatal day 3 . On postnatal day 5, pups were ip-injected with i) PRE-084 $1 \mu \mathrm{g} / \mathrm{g}$ bodyweight or ii) vehicle and were subsequently subjected to either i) hyperoxia ( $\mathrm{HX}, \mathrm{FiO} 2>0.9$ ) or ii) normoxia (NX, FiO2=0.21) for 24 hours. At the end of exposure, animals were sacrificed and brains were processed for caspase-3 analysis using immunohistochemistry and Western Blotting.

Results A single LPS injection significantly increased the number of activated caspase-3-positive cells in cortical grey matter after hyperoxic exposure, which was reduced by PRE-084 administration (mean number of cells \pm SEM; LPS_NX_vehicle $31.26 \pm 1.29$ vs. LPS HX_vehicle $38.11 \pm 1.13$, p $<0.01$ vs. LPS_HX_PRE-084 33.66 \pm 1.54 , $\mathrm{p}<0.05 ; \mathrm{n}=6-7)$. Western Blot analyses showed a strong reduction in caspase-3 cleavage in PRE-084-treated pups compared to vehicleinjected controls in both pre-sensitized and non-pre-sensitized animals after hyperoxic exposure.

Conclusion PRE-084 reduces inflammation-sensitized hyperoxiainduced injury in the developing rat brain by inhibition of apoptosis. Sigma agonists are a potential therapeutic approach in perinatal brain injury and merit further studies.

\section{ENDOTHELIAL DYSFUNCTION AND PERINATAL MORTALITY OF PRETERM INFANTS EXPOSURED INTRAUTERINE HYPOXIA}

doi:10.1136/archdischild-2012-302724.1084

PA Orujova, ${ }^{2} \mathrm{HA}$ Saadat, ${ }^{3} \mathrm{H}$ Safikhan, ${ }^{3} \mathrm{~A}$ Saadat, ${ }^{2} \mathrm{M}$ Sevinj, ${ }^{4} \mathrm{M}$ Tarana. ${ }^{1}$ Azerbailan Medical University; ${ }^{2}$ Neonatology; ${ }^{3}$ Azerbaijan Medical University; ${ }^{4}$ Maternity Hospital, Baku, Azerbaijan

Aim To investigate the endothelial dysfunction in preterm newborns with birth weigth over 1500 gr which died in early neonatal period.

Methods For this purpose 30 surviving and 15 dead newborns with birth weight over1500gr were examined and divided in two groups: control group included 30 newborns, main group- 15 infants died in early neonatal period. İn all infants were determined antenatal hypoxia by ultrasonography examination. In order to determine endothelial dysfunction sİCAM-1 and sVCAM-1 concentrations were detected by Uscn (Life Science Inc., USA) kits in 1st-3rd and 5th-7 th days. The Student $t$-test and the Mann-Whitney test were used for comparison of parametric and non-parametric parameters. Results On the 1st-3rd day the levels of the both adhesion molecules were higher in main group than the control group, but on the 5 th-7th days of life they were significantly decreased in comparison with either control group and 1st-3rd days parameters. Adhesion molecules concentations in control group were increased in dynamic $(\mathrm{p}<0.01)$.

Conclusion The appointment of the level of adhesion molecules may give an opportunity to determine the endothelial dysfunction and may be predict about perinatal outcome.

\section{CARDIOVASCULAR DYSFUNCTION IN INFANTS WITH NEONATAL ENCEPHALOPATHY IN THE 1ST WEEK OF LIFE}

doi:10.1136/archdischild-2012-302724.1085

1,2,3 K Armstrong, ${ }^{1,2} \mathrm{D}$ Sweetman, ${ }^{3} \mathrm{O}$ Franklin, ${ }^{1,2,4} \mathrm{E}$ Molloy. ${ }^{1}$ National Children's Research Centre, National Children's Research Centre, ${ }^{2}$ Neonatology, National Maternity Hospital; ${ }^{3}$ Paediatric Cardiology, Our Lady's Children's Hospital; " Paediatrics, Royal College of Surgeons, Dublin, Ireland

Background and Aims Perinatal asphyxia may result in transient myocardial ischameia, confirmed by elevated Troponin $\mathrm{T}$ levels Gold standard echocardiographic measures of contractility (ejection and shortening fraction) may not pick up subtle ischaemic changes. Tissue Doppler imaging (TDI) allows assessment of systolic and diastolic function. Used in conjunction with Troponin T TDI may offer superior measure of myocardial contractility.

Methods Term infants with evidence of Neonatal Encephalopathy (NE) underwent echocardiography on Day 1 \& 7 of life. Healthy term controls had one echocardiogram on Day 1. Serum Troponin T levels were recorded in infants with NE. Myocardial velocities were obtained using a pulsed wave doppler from an apical four chamber view. Peak systolic (S'), early diastolic (E') and late diastolic (A') velocities were recorded.

Results 17 patients with evidence of $\mathrm{NE}$ and 20 term controls were recruited. Mean birthweight (SD) was $3.6 \mathrm{~kg}(0.9)$ and gestation 39 (5) weeks. TDI systolic and diastolic velocities increased between Day $1 \& 7$ in infants with NE. All day 1 measures in the NE group were less than the controls. There was no significant difference between the shortening/ejection fraction on day 1 between the two groups (NE: 33.7-35.3\%; Control: 64.3-67.4\%) Troponin levels were significantly elevated on Day 1 compared to Day 7 in NE group $(\mathrm{p}<0.05)(0.53-0.38 \mathrm{ng} / \mathrm{ml})$

Conclusions TDI measures in infants with NE are less than controls on Day 1. Troponin levels were initially significantly increased providing further evidence of myocardial ischaemia in infants with NE.

\section{6 SEIZURES ARE ASSOCIATED WITH ALTERED HIPPOCAMPAL DIFFUSION IN NEONATES WITH HYPOXIC-ISCHEMIC ENCEPHALOPATHY}

doi:10.1136/archdischild-2012-302724.1086

${ }^{1} \mathrm{JG}$ Feenstra, ${ }^{2} \mathrm{PN}$ Lee, ${ }^{2} \mathrm{D} \mathrm{Xu},{ }^{3} \mathrm{SL}$ Bonifacio, ${ }^{4} \mathrm{DM}$ Ferriero, ${ }^{2} \mathrm{AJ}$ Barkovich, ${ }^{4} \mathrm{HC}$ Glass. 'University of Groningen, Groningen, The Netherlands, ${ }^{2}$ Radiology \& Biomedical Imaging ${ }^{3}$ Pediatrics; ${ }^{4}$ Neurology \& Pediatrics, University of California, San Francisco, CA, USA

In animal models, neonatal seizures (NS) alter hippocampal development and lead to long-term deficits. Whether NS similarly affect humans is not known. The goal of this study was to assess whether NS are associated with altered hippocampal microstructure in neonates with hypoxic ischemic encephalopathy.

We included 6 neonates with and 27 without seizures. All were treated with therapeutic hypothermia after birth. Neonatal (median 5 days) and 6-month diffusion tensor imaging was used to measure apparent diffusion coefficient (ADC) from regions of interest (ROIs) in the hippocampus, basal ganglia, thalamus and frontal white matter.

ADC was significantly lower on the 6-month scan as compared to the neonatal scan for all ROIs. There were no significant differences in $\mathrm{ADC}$ on the early scan when comparing neonates with and without seizures. At 6 months, infants with seizures as neonates had a $6 \%$ higher hippocampal ADC (95\% confidence interval: $0-11 \%, \mathrm{p}<0.05)$. There was no significant difference in ADC for the other ROIs.

These preliminary results suggest that NS are associated with altered hippocampal structural development. Because the difference was seen only in the hippocampus, and on follow-up imaging but 